\title{
Aplicabilidade do processo de territorialização como estratégica para o desenvolvimento da promoção da saúde na Atenção Básica
}

\author{
Applicability of the territorialization process as a strategy for the development of health promotion \\ in Primary Care
}

Aplicabilidad del proceso de territorialización como estrategia para el desarrollo de la promoción de la salud en Atención Primaria

Recebido: 14/07/2021 | Revisado: 20/07/2021 | Aceito: 04/08/2021 | Publicado: 09/08/2021

\author{
Jordeilson Luis Araujo Silva \\ ORCID: https://orcid.org/0000-0002-2806-0377 \\ Programa de Pós-Graduação em Saúde da Família Sobral, Brasil \\ E-mail: jordeilsonluis@gmail.com \\ Lidyane Parente Arruda \\ ORCID: https://orcid.org/0000-0002-5218-1259 \\ Programa de Pós-Graduação em Saúde da Família Sobral, Brasil \\ E-mail: lidyaneparente@hotmail.com \\ Roberlandia Evangelista Lopes \\ ORCID: https://orcid.org/0000-0002-6810-7416 \\ Programa de Pós-Graduação em Saúde da Família Sobral, Brasil \\ E-mail: roberlandialopes@hotmail.com \\ Fernando Daniel de Oliveira Mayorga \\ ORCID: https://orcid.org/0000-0002-8488-5234 \\ Programa de Pós-Graduação em Saúde da Família Sobral, Brasil \\ E-mail: mayorga@sobral.ufc.br \\ George Ventura Alves Neri \\ ORCID: https://orcid.org/0000-0002-8378-655X \\ Fundação Oswaldo Cruz, Brasil \\ E-mail: georgeventura@ifma.edu.br
}

\begin{abstract}
Resumo
Os conceitos de território e de territorialização são problematizados, com base em concepções diversas, a fim de dialogarem com as atribuições e desafios da Vigilância em Saúde e, especialmente O objetivo do artigo é analisar na literatura a importância e aplicabilidade do processo de territorialização como estratégica para o desenvolvimento da promoção da saúde na Atenção Básica. Trata-se de uma revisão integrativa de abordagem qualitativa realizada no mês de julho de 2021, através das bases de dados da Biblioteca Virtual de Saúde (BVS). Para a formulação da questão de pesquisa, utilizou-se a estratégia PICo. Partir da vigilância do território pede-se realizar o planejamento de ações promoções e prevenção das vulnerabilidades. Com isso, a territorialização constitui instrumento de organização dos processos de trabalho e das práticas de saúde. Proporcionaram informação mínima dos sistemas usados, contribuindo mesmo tenha dificuldade desenvolver o seu papel na vigilância do território. Portanto, considera-se que a o processo de territorialização deve-se reconhecer as percepções e condições de vida para propor ações de promoção de saúde na comunidade.
\end{abstract}

Palavras-chave: Atenção Primária à Saúde; Territorialização; Promoção da saúde.

\begin{abstract}
The concepts of territory and territorialization are problematized, based on different conceptions, in order to dialogue with the attributions and challenges of Health Surveillance and, especially. The objective of the article is to analyze in the literature the importance and applicability of the territorialization process as a strategic for the development of health promotion in Primary Care. This is an integrative review of a qualitative approach carried out in July 2021, through the Virtual Health Library (VHL) databases. To formulate the research question, the PICo strategy was used. From the surveillance of the territory, it is necessary to carry out the planning of actions, promotions and prevention of vulnerabilities. Thus, territorialization is an instrument for organizing work processes and health practices. They provided minimal information on the systems used, contributing even if it was difficult to develop their role in the surveillance of the territory. Therefore, it is considered that the territorialization process must recognize the perceptions and living conditions in order to propose actions to promote health in the community.
\end{abstract}

Keywords: Primary Health Care; Territorialization; Health promotion. 


\begin{abstract}
Resumen
Se problematizan los conceptos de territorio y territorialización, a partir de distintas concepciones, con el fin de dialogar con las atribuciones y desafíos de la Vigilancia en Salud y, en especial, para el desarrollo de la promoción de la salud en Atención Primaria. Se trata de una revisión integradora de un enfoque cualitativo realizado en julio de 2021, a través de las bases de datos de la Biblioteca Virtual en Salud (BVS). Para formular la pregunta de investigación se utilizó la estrategia PICo. Desde la vigilancia del territorio, es necesario realizar la planificación de acciones, promociones y prevención de vulnerabilidades. Así, la territorialización es un instrumento para organizar los procesos de trabajo y las prácticas de salud. Proporcionaron información mínima sobre los sistemas utilizados, contribuyendo incluso si era difícil desarrollar su papel en la vigilancia del territorio. Por tanto, se considera que el proceso de territorialización debe reconocer las percepciones y condiciones de vida para proponer acciones de promoción de la salud en la comunidad.

Palabras clave: Primeros Auxilios; Territorialización; Promoción de la salud.
\end{abstract}

\title{
1. Introdução
}

A territorialização é uma política fundamental atenção básica do Brasil. Com isso, constitui em um importante instrumento de organização dos processos de trabalho e das práticas de saúde, posto que as ações de saúde são implementadas sobre uma base territorial detentora de uma delimitação espacial previamente determinada (Faria, 2020).

Tentar compreender o território é, em princípio, assumir a atitude de percorrer diversas e distintas acepções elaboradas acerca dele ao longo dos anos. Inúmeros autores, filiados às mais diferentes tradições intelectuais e correntes de pensamento, intentaram realizar esse percurso, e cada qual, ao seu modo e no seu tempo, contribuiu para a compreensão do mesmo (Santos \& Raquel, 2011).

Desta forma, observamos que o território em saúde tem a sua particularidade, que determina diferentes perfis demográficos, epidemiológicos, econômicos, sociais e culturais, os quais apresenta uma em constante transformação. Com isso, a performance das equipes de saúde nos territórios buscar considerar essas particularidades (Pessoa et al., 2013).

No Brasil, a estruturação da política de Saúde vem sofrendo um processo de sucessivas mudanças, com a atuação de importantes segmentos sociais e políticos no sentido de reivindicar o avanço do movimento pela Reforma Sanitária Com isso, lugar de entendimento do processo de adoecimento, em que as representações sociais do processo saúde-doença envolvem as relações sociais e as significações culturais (Justo et al., 2017).

O processo de territórização, no contexto do sistema de saúde brasileiro, tem definições e princípio descrito na Lei Orgânica da Saúde (Lei 8.080) deliberou que o município passaria a ser, de fato, o responsável imediato pelo atendimento das necessidades e demandas de saúde da sua população e das exigências de intervenções saneadoras em seu território (Brasil, 1990).

A Estratégia Saúde da Família (ESF) compreende uma reorientação de práticas nesse contexto, que se estrutura desde a atuação de equipes multiprofissionais em um território determinado desenvolvendo ações a partir do conhecimento e vivências da realidade local bem como das necessidades da comunidade (Nonato et al., 2020).

Neste sentido, as equipes de Saúde da Família devem organizar para atender a uma sua população, com isso, sendo protagonista na responsabilidade sanitária, atendendo a dinamicidade existente no território em que vive essa população (Brasil, 2012).

Assim, torna-se prioritário reconhecer o território como um espaço social onde a sociedade se constrói e se reconstrói, no cerne de seu processo de trabalho e produção; dividindo-se em classes diferenciadas, com acessos também desiguais aos bens de consumo, incluídos os serviços de saúde.

O interesse em pesquisar o tema escolhido, surgiu da necessidade em conhecer melhor a realidade o processo de territorialização pela atenção básica existente na literatura. Assim, a territorialização é como elemento importante e estratégico nesse processo de ampliação da assistência em saúde.

Com isso, a estrutura social, política e econômica que constituem o cenário mundial, cada vez mais exigem das autoridades e da sociedade a aplicação de estratégias e ações positivas do fortalecimento da territorialização. Baseando-se no 
objeto e problemática do estudo, torna-se importante buscar respostas para a seguinte questão que norteia a presente pesquisa: Quais as evidências científicas disponíveis na importância e aplicabilidade do processo de territorialização como estratégica para o desenvolvimento da promoção da saúde na Atenção Primária de Saúde? O objetivo do artigo é analisar na literatura a importância e aplicabilidade do processo de territorialização como estratégica para o desenvolvimento da promoção da saúde na Atenção Básica.

\section{Metodologia}

\subsection{Delineamento do Estudo}

Trata-se de pesquisa qualitativa descritiva, uma revisão integrativa da literatura utilizando a estratégia PICo, elaborada a partir das seguintes etapas definição do tema e formulação dos objetivos e da questão norteadora; busca na literatura e delimitação para a inclusão dos estudos; categorização dos estudos; avaliação dos estudos; interpretação dos resultados e apresentação da revisão/síntese do conhecimento (Mendes et al., 2019).

Desta forma, a revisão integrativa, permite a síntese de diferentes estudos publicados que possibilita resultados a respeito do tema estudado. É um método que possibilita a leitura de todo o conhecimento científico disponível (Souza, 2010).

\subsection{Período e Local do Estudo}

A pesquisa ocorreu no mês junho de 2021, nas bases de dados da Literatura Latino-Americana e do Caribe em Ciências da Saúde (LILACS), Base de Dados em Enfermagem (BDENF) via Biblioteca Virtual em Saúde (BVS), da National Library of Medicine (MEDLINE) via PubMed e Scientific Electronic Library Online (SCIELO).

O estudo foi realizado, utilizando bases eletrônicas, definidas por critérios de inclusão e de exclusão, das publicações. De tal modo, foram excluídos: cartas ao editor, anais de eventos científicos, teses, dissertações e revisões de literatura. Os artigos duplicados foram contabilizados apenas uma única vez e serão selecionadas as publicações que estiveram em concordância com a pergunta norteadora.

Para a formulação da questão de pesquisa, utilizou-se a estratégia PICo (P - população; I - intervenção/interesse; Co contexto (Mendes et al, 2019). Deste modo, considerou-se (P) Atenção Primária de Saúde; (I) Territorialização; e (Co) Promoção da saúde. Assim, a questão norteadora elaborada foi: Quais as evidências científicas disponíveis na importância e aplicabilidade do processo de territorialização como estratégica para o desenvolvimento da promoção da saúde na Atenção Primária de Saúde?

Desta forma, utilizaram-se os descritores controlados encontrados nos Descritores em Ciência da Saúde (DeCS), identificando-se, assim os seguintes descritores: Pessoal da Saúde; Contaminação de Medicamentos; Serviços de Saúde. Os descritores não controlados (palavras-chave) foram utilizados como sinônimos no idioma inglês e português.

Para sistematizar a coleta da amostra, utilizou-se o recurso de busca avançada, respeitando particularidades e características distintas de cada base de dados. Os descritores foram combinados entre si com o operador booleano OR, dentro de cada conjunto de termos da estratégia PICo e, por sequência, cruzados com o operador booleano AND.

Adotaram-se os seguintes critérios de inclusão para os estudos: artigos originais completos que abordam o processo de territorialização no desenvolvimento da atenção primaria publicados no período nos últimos 05 anos, escritos nas línguas portuguesa, inglesa e espanhola.

Desta forma, foram encontrados no total de 118 artigos, sendo do LILACS: 85 artigos, na BDENF:21 e MEDLINE =12 artigos. Deste modo, os artigos foram analisados pelos seguintes critérios. Foram realizadas leituras críticas dos títulos e resumos dos artigos selecionados, com a finalidade de verificar a adequabilidade de acordo com a questão norteadora, assim, aplica os critérios de inclusão e exclusão apresentados, (Figura 2). Assim, a amostra final de artigos foram de 4 artigos. Para sistematizar os artigos, foi adaptado um instrumento de coleta de Mendes, Silva e Galvão (2008) com os seguintes parâmetros: autores, ano 
da publicação, bases de dados, título, metodologia do estudo, revistas periódicos e nível de evidência.

Os níveis de evidência utilizados para a classificação foram baseados conforme publicação na RBO: Nível I: Metanálise de múltiplos estudos controlados; Nível II: estudos experimentais individuais; Nível III: estudos como ensaio clínico não randomizado; Nível IV: descrição de série de casos, com análise de resultados, sem estudo comparativo; Nível V: dados de avaliação de programa de dados obtidos de forma sistemática (Camanho, 2009).

Figura 1: Síntese dos estudos incluídos na revisão integrativa. Timon, MA, Brasil, 2021.

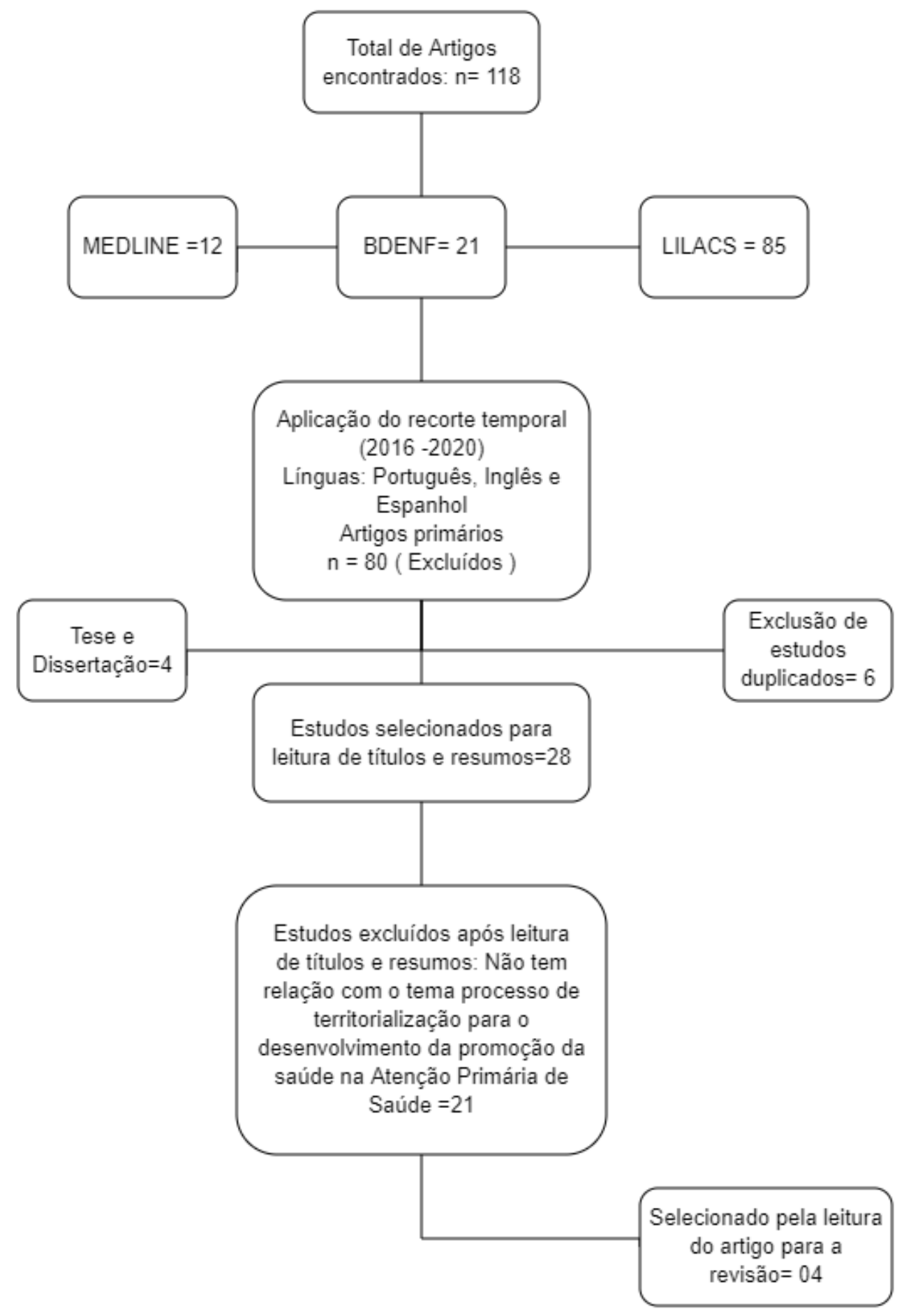

Fonte: Autores. 


\subsection{Análise dos Dados}

Serão extraídos os dados secundários das publicações selecionadas por meio objetivos, o tipo de estudo, os resultados e as conclusões, para assegurar que a totalidade seja extraída, para minimizar os erros na transcrição, para garantir a precisão da checagem das informações e para servir como registros (Mendes et al., 2008).

Os dados foram organizados e analisados com o auxílio do software Excel, e apresentados por meio de estatística descritiva (frequência absoluta e relativa). Serão elaborados sínteses e recortes dos artigos selecionados para a construção do texto final.

Como conclusão desta revisão integrativa, será realizada uma elaboração síntese das evidências disponíveis, com a produção dos resultados da análise do conhecimento apresentado nos resultados.

\section{Resultados e Discussão}

Nesta revisão foram selecionados 05 artigos, dos quais três (60\%) foi identificado na SCIELO, o um (20\%) na BDENF, um (20\%) MEDLINE. Desses, dois (40\%) tinham sido publicados em periódicos de enfermagem, três (60\%) em revistas interdisciplinares de saúde.

No que tange ao desenho dos estudos, 3 (60\%) pesquisa com metodologia transversal, 1 (20\%) relato de experiência, e um (20\%) Pesquisa qualitativa, perspectiva crítico-reflexiva. Quanto ao nível de evidência, cem (100\%) publicações foram classificadas com o IV.

Quadro 1 - Síntese dos estudos primários incluídos na pesquisa. Timon, MA, 2021.

\begin{tabular}{|c|c|c|c|c|c|}
\hline $\begin{array}{c}\text { Autores/ Ano/ } \\
\text { Local }\end{array}$ & Objetivo & $\begin{array}{c}\text { Bases de } \\
\text { dados }\end{array}$ & $\begin{array}{c}\text { Delineamento } \\
\text { do Estudo }\end{array}$ & $\begin{array}{l}\text { Principais } \\
\text { resultados }\end{array}$ & $\begin{array}{c}\text { Nível de } \\
\text { Evidência }\end{array}$ \\
\hline $\begin{array}{l}\text { (Souza et al., } \\
\text { 2019) } \\
\text { Chapecó }\end{array}$ & $\begin{array}{c}\text { Relatar a experiência de quatro } \\
\text { estudantes do curso de medicina de } \\
\text { uma universidade pública do Sul do } \\
\text { Brasil, no componente curricular } \\
\text { Saúde Coletiva II, durante as } \\
\text { vivências realizadas em um Centro de } \\
\text { Saúde da Família (CSF). }\end{array}$ & SCIELO & $\begin{array}{c}\text { relato de } \\
\text { experiência. }\end{array}$ & $\begin{array}{c}\text { Território permite conhecer as } \\
\text { características da população, pois a } \\
\text { base espacial torna-se recurso da } \\
\text { territorialização, permitindo o } \\
\text { levantamento de dados sobre a } \\
\text { população e seus problemas de saúde, } \\
\text { bem como sobre o impacto dos } \\
\text { serviços de saúde }\end{array}$ & IV \\
\hline $\begin{array}{c}\text { (Pedebos et al. } \\
\text { 2020) }\end{array}$ & $\begin{array}{l}\text { identificar fragilidades no processo } \\
\text { de trabalho dos Agentes Comunitários } \\
\text { de Saúde (ACS) e a percepção de } \\
\text { importância dada por eles sobre as } \\
\text { ações em vigilância do território para } \\
\text { o trabalho da equipe de saúde, } \\
\text { juntamente a um processo de } \\
\text { sensibilização e capacitação dos ACS } \\
\text { sobre a temática. }\end{array}$ & SCIELO & $\begin{array}{c}\text { Estudo } \\
\text { transversal. }\end{array}$ & $\begin{array}{l}\text { Importância do seu trabalho de } \\
\text { vigilância do território para o } \\
\text { planejamento das ações de sua } \\
\text { respectiva equipe e, } \\
\text { consequentemente, para a } \\
\text { comunidade, compreendendo que seu } \\
\text { trabalho não se resume a identificar } \\
\text { problemas no território, mas, também, } \\
\text { orientar a população para a prevenção } \\
\text { de doenças. }\end{array}$ & IV \\
\hline $\begin{array}{l}\text { (Nonato et al., } \\
\text { 2020) }\end{array}$ & $\begin{array}{c}\text { identificar as estratégias de } \\
\text { gerenciamento utilizadas pelas } \\
\text { equipes de ESF de uma Unidade } \\
\text { Básica de Saúde na organização do } \\
\text { trabalho em um território de } \\
\text { vulnerabilidade social exposto à }\end{array}$ & SCIELO & $\begin{array}{c}\text { Estudo } \\
\text { transversal }\end{array}$ & $\begin{array}{c}\text { Territórios onde equipes de APS } \\
\text { atuam e, para seu enfrentamento neste } \\
\text { contexto faz-se necessária uma ação } \\
\text { multiprofissional e interdisciplinar na } \\
\text { elaboração de estratégias de } \\
\text { prevenção à violência. }\end{array}$ & IV \\
\hline
\end{tabular}


Research, Society and Development, v. 10, n. 10, e221101018283, 2021

(CC BY 4.0) | ISSN 2525-3409 | DOI: http://dx.doi.org/10.33448/rsd-v10i10.18283

\begin{tabular}{|c|c|c|c|c|c|}
\hline & violência.. & & & & \\
\hline $\begin{array}{l}\text { (Pinto et al., } \\
\text { 2017) }\end{array}$ & $\begin{array}{l}\text { Compreender as demandas cotidianas } \\
\text { da Estratégia Saúde da Família (ESF) } \\
\text { na prática clínica da equipe e as } \\
\text { vulnerabilidades sociais do território } \\
\text { comunitário urbano. }\end{array}$ & MEDLINE & $\begin{array}{l}\text { Pesquisa } \\
\text { qualitativa, } \\
\text { perspectiva } \\
\text { crítico- } \\
\text { reflexiva. }\end{array}$ & $\begin{array}{l}\text { Os trabalhadores não cumprem os } \\
\text { passos do descarte correto. A maioria } \\
\text { desconhece a legislação vigente. Os } \\
\text { profissionais identificam a } \\
\text { contaminação do ambiente, uso } \\
\text { indevido dos medicamentos } \\
\text { descartados incorretamente e } \\
\text { resistência bacteriana aos } \\
\text { medicamentos como principais } \\
\text { consequências do descarte icorreto. }\end{array}$ & IV \\
\hline $\begin{array}{l}\text { (Souza et al., } \\
\text { 2019) } \\
\text { Chapecó }\end{array}$ & $\begin{array}{c}\text { Relatar a experiência de quatro } \\
\text { estudantes do curso de medicina de } \\
\text { uma universidade pública do Sul do } \\
\text { Brasil, no componente curricular } \\
\text { Saúde Coletiva II, durante as } \\
\text { vivências realizadas em um Centro de } \\
\text { Saúde da Família (CSF). }\end{array}$ & SCIELO & $\begin{array}{c}\text { relato de } \\
\text { experiência. }\end{array}$ & $\begin{array}{l}\text { Território permite conhecer as } \\
\text { características da população, pois a } \\
\text { base espacial torna-se recurso da } \\
\text { territorialização, permitindo o } \\
\text { levantamento de dados sobre a } \\
\text { população e seus problemas de saúde, } \\
\text { bem como sobre o impacto dos } \\
\text { serviços de saúde }\end{array}$ & IV \\
\hline $\begin{array}{l}\text { ( Pedebos et } \\
\text { al. 2020) }\end{array}$ & $\begin{array}{l}\text { identificar fragilidades no processo } \\
\text { de trabalho dos Agentes Comunitários } \\
\text { de Saúde (ACS) e a percepção de } \\
\text { importância dada por eles sobre as } \\
\text { ações em vigilância do território para } \\
\text { o trabalho da equipe de saúde, } \\
\text { juntamente a um processo de } \\
\text { sensibilização e capacitação dos ACS } \\
\text { sobre a temática. }\end{array}$ & SCIELO & $\begin{array}{c}\text { Estudo } \\
\text { transversal. }\end{array}$ & $\begin{array}{c}\text { Importância do seu trabalho de } \\
\text { vigilância do território para o } \\
\text { planejamento das ações de sua } \\
\text { respectiva equipe e, } \\
\text { consequentemente, para a } \\
\text { comunidade, compreendendo que seu } \\
\text { trabalho não se resume a identificar } \\
\text { problemas no território, mas, também, } \\
\text { orientar a população para a prevenção } \\
\text { de doenças. }\end{array}$ & IV \\
\hline $\begin{array}{l}\text { (Nonato et al., } \\
2020)\end{array}$ & $\begin{array}{c}\text { identificar as estratégias de } \\
\text { gerenciamento utilizadas pelas } \\
\text { equipes de ESF de uma Unidade } \\
\text { Básica de Saúde na organização do } \\
\text { trabalho em um território de } \\
\text { vulnerabilidade social exposto à } \\
\text { violência.. }\end{array}$ & SCIELO & $\begin{array}{c}\text { Estudo } \\
\text { transversal }\end{array}$ & $\begin{array}{c}\text { Territórios onde equipes de APS } \\
\text { atuam e, para seu enfrentamento neste } \\
\text { contexto faz-se necessária uma ação } \\
\text { multiprofissional e interdisciplinar na } \\
\text { elaboração de estratégias de } \\
\text { prevenção à violência. }\end{array}$ & IV \\
\hline $\begin{array}{l}\text { (Pinto et al., } \\
\text { 2017) }\end{array}$ & $\begin{array}{l}\text { Compreender as demandas cotidianas } \\
\text { da Estratégia Saúde da Família (ESF) } \\
\text { na prática clínica da equipe e as } \\
\text { vulnerabilidades sociais do território } \\
\text { comunitário urbano. }\end{array}$ & MEDLINE & $\begin{array}{l}\text { Pesquisa } \\
\text { qualitativa, } \\
\text { perspectiva } \\
\text { crítico- } \\
\text { reflexiva. }\end{array}$ & $\begin{array}{l}\text { Os trabalhadores não cumprem os } \\
\text { passos do descarte correto. A maioria } \\
\text { desconhece a legislação vigente. Os } \\
\text { profissionais identificam a } \\
\text { contaminação do ambiente, uso } \\
\text { indevido dos medicamentos } \\
\text { descartados incorretamente e } \\
\text { resistência bacteriana aos } \\
\text { medicamentos como principais } \\
\text { consequências do descarte icorreto. }\end{array}$ & IV \\
\hline
\end{tabular}

Fonte: Autores. 
A primeira entrada do usuário do Sistema Único de Saúde (SUS) é a Atenção Primária à Saúde (APS) a principal porta de entrada da Rede de Atenção à Saúde (RAS), com a sua principal característica é organiza e ordenar o cuidado dos pacientes nas redes de atenção. Com isso, o Brasil é um dos países que constitui da ESF como planejamento estratégico de expansão e consolidação no território nacional (Brasil, 2017).

A ESF possui como princípios norteadores que buscam atende as necessidades de saúde da população, proporcionando ações de promoção, prevenção, recuperação da saúde, bem como ações reabilitação de doenças e vigilância de agravos mais frequentes. Desta forma as necessidades em ter acesso aos serviços de saúde podem ser minimizadas quando inseridas e desenvolvidas nos territórios (Justo et al., 2017).

Ao se apropriar do território e das dinâmicas que nele se estabelecem, os profissionais de saúde têm melhores condições de compreender algumas escolhas que, circunstancialmente, as pessoas fazem, em função do acesso que têm a determinadas estruturas sociais. Assim como as pessoas não escolhem viver em más condições, elas não escolhem ter menor grau de escolaridade, muito menos escolhem ficar doentes (Pedebos et al., 2018).

A delimitação de uma área de atuação de uma ESF compreende um dos sentidos da territorialização. No entanto, o desafio é procurar qualificar esse uso com ênfase no reconhecimento do ambiente, da população, do acesso às ações e serviços de saúde e da dinâmica social existente em cada território. Nesse sentido, a territorialização se coloca como um meio capaz de produzir mudanças no modelo assistencial e nas práticas sanitárias vigentes (Santos \& Lima, 2020).

Sabe-se que a troca de saberes possibilita uma construção analítica da distribuição de agravos na saúde e da compreensão dos múltiplos fatores implicados nas dinâmicas territoriais. Dessa forma, fomenta a busca por estratégias responsivas às especificidades de cada local e por modelos que visem a melhoria da qualidade de vida, com ações de promoção da saúde e prevenção de doenças e agravos, sendo de fundamental importância o aporte da geografia da saúde nessa construção (Souza et al., 2019).

O território, longe de ser apenas um espaço político operativo do sistema de saúde, é o local onde ocorre a interação população-serviço, caracterizado por uma comunidade específica que habita determinada área e apresenta problemas de saúde específicos (Colussi \& Pereira, 2016).

O território é advém do processo histórico, político e social que influencia o modo como as pessoas vivem e, bem como, aparte das necessidades do território podemos organizar e realizar o planejamento das ações de promoção em a serem desenvolvida pelas equipes de ESF (Nonato et al., 2020).

Por manter uma grande extensão territorial, as atividades executadas ainda se mantêm centralizadas nos dispositivos de interação social instalados em bairros mais centrais dessa região (Pinto et al., 2017).

Diante de um território de elevada vulnerabilidade, o cuidado prestado à população parece obedecer a racionalidades que convergem para o assistencialismo, em ações emergenciais voltadas à resolução imediata de problemas agudos a partir da lógica queixa-diagnóstico-conduta, reforçando o modelo assistencial pautado predominantemente em ações de tratamento (Souza et al., 2019).

\section{Conclusão}

Nesse sentido, é essencial o conhecimento interdisciplinar sobre determinantes e condicionantes sociais e ambientais. Esse envolvimento permitiu que as ações de vigilância em saúde desenvolvidas na Atenção Primária à Saúde estivessem alicerçadas na consciencialização sobre a importância do território, papel desempenhado diariamente pelo profissional médico no contexto dos atendimentos de saúde.

Foi evidenciado que mesmo sem apoio de políticas públicas específicas a essas situações, os profissionais desenvolvem estratégias que incluem o fortalecimento da equipe como forma de proteção coletiva, acolhimento focado no atendimento integral 
e vínculo. A população é aliada ao facilitar o acesso ao atendimento de vulneráveis e alerta os profissionais das situações críticas no território.

\section{Referências}

Brasil. (1990). Lei 8080 de 19 de setembro de 1990. Dispõe sobre as condições para a promoção, proteção e recuperação da saúde, a organização e o funcionamento dos serviços correspondentes e dá outras providências. http://www.planalto.gov.br/ccivil_03/leis/18080.htm

Brasil. (2017). Ministério da Saúde. Portaria n. 2.436, de 21 de setembro de 2017. Aprova a Política Nacional de Atenção Básica, estabelecendo a revisão de diretrizes para a organização da Atenção Básica, no âmbito do Sistema Único de Saúde. 2017 . http://bvsms.saude.gov.br/bv s/saudelegis/gm/2017/prt2436_22_09_2017.html

Camanho, G. L. (2009). Nível de evidência. Rev. bras. Ortop.

Colussi, C. F., \& Pereira, K. G. (2016). Territorialização como instrumento do planejamento local na atenção básica. Dicionário da Educação Profissional em Saúde. https://ares.unasus.gov.br/acervo/html/ARES/13957/1/TERRITORIALIZACAO_LIVRO.pd

Rivaldo, M. d. F. (2013). A Territorialização Da Atenção Primária À Saúde No Sistema Único De Saúde E A Construção De Uma Perspectiva De Adequação Dos Serviços Aos Perfis Do Território1. Revista Brasileira de Geografia Médica e da Saúde. https://edisciplinas.usp.br/pluginfile. php/4188196/mod_resource/content/1/A\%20TERRITORIALIZA\%C3\%87\%C3\%83O\%20DA\%20ATEN\%C3\%87\%C3\%83O\%20PRIM\%C3\%81RIA.pdf.

Justo, L. G., Severo, A. K. d. S., Silva, A. V. F., Soares, L. S., Júnior, F. L. e. S., \& Pedrosa, J. I. d. S. (n.d.). A territorialização na Atenção Básica: um relato de experiência na formação médica. Comunicação, Saúde, Educação. https://www.scielo.br/j/icse/a/G4rRVvny64R7HM9zfHkHQcc/?lang=pt\#.

Koga, D. (2013). Aproximações sobre o conceito de território e sua relação com a universalidade das políticas sociais. Serviço Social em Revista. https://doi.org/10.5433/1679-4842.2013v16n1p30.

Mendes, K. D. S., Silveira, R. C. d. C. P., \& Galvão, C. M. (2008). Revisão Integrativa: Método De Pesquisa Para A Incorporação De Evidências Na Saúde E Na Enfermagem. Texto Contexto Enferm. https://www.scielo.br/j/tce/a/XzFkq6tjWs4wHNqNjKJLkXQ/?format=pdf\&lang=pt

Mendes, K. D. S., Silveira, R. C. d. C. P., \& Galvão, C. M. (2019). Use Of The Bibliographic Reference Manager In The Selection Of Primary Studies In Integrative Reviews. Texto \& Contexto - Enfermagem. https://www.scielo.br/j/tce/a/HZD4WwnbqL8t7YZpdWSjypj/?lang=en\#ModalArticles

Faria, R. M. d. (2020). A territorialização da Atenção Básica à Saúde do Sistema Único de Saúde do Brasil. Ciênc. Saúde coletiva. https://www.scielo.br/j/csc/a/jSZ7b65YpPSTwLfYWpRhg5z/?lang=pt

Nonato, L. O. F., Peres, A. M., Khalaf, D. K., Souza, M. A. R. d., Figueiredo, K. C., \& Lapierre, J. (2020). Estratégias de gerenciamento na Atenção Primária à Saúde em territórios de vulnerabilidade social expostos à violência. Rev Esc Enferm. http://www.revenf.bvs.br/pdf/reeusp/v54/1980-220X-reeusp-54-e03608.pdf

Pedebos, L. A., Rocha, D. K., \& Tomasi, Y. (2018). A vigilância do território na atenção primária: contribuição do agente comunitário na continuidade do cuidado. Saúde debate. https://www.scielosp.org/article/sdeb/2018.v42n119/940-951/\#

Pessoa, V. M., Rigotto, R. M., Carneiro, F. F., \& Teixeira, A. C. d. A. (2013). Sentidos e métodos de territorialização na atenção primária à saúde. Ciênc. saúde coletiva. https://www.scielo.br/j/csc/a/mHwc3y7WHkVF6tGb7k8JS3J/?lang=pt

Pinto, A. G. A., Jorge, M. S. B., Marinho, M. N. A. d. S. B., Vida, E. C. F., Aquino, P. d. S., \& Vidal, E. C. F. (2017). Experiences in the Family Health Strategy: demands and vulnerabilities in the territory. Rev Bras Enferm. https://www.scielo.br/j/reben/a/DzsdQGHvZn86SWzhVdKmNLM/?format=pdf

Santos, M. A. M. d., \& Lima, F. M. d. (2020). A Territorialização E A Integração Ensino-Serviço Na Enfermagem: Um Relato De Experiência Sob A Ótica Dos Egressos. Tema em Saúde. https://temasemsaude.com/wp-content/uploads/2020/06/20311.pdf

Santos, A. L., \& Rigotto, R. M. (2010). Território e territorialização: incorporando as relações produção, trabalho, ambiente e saúde na atenção básica à saúde. https://www.scielo.br/j/tes/a/H5BtBJTGvQZgSXKvNrTKphp/?lang=pt

Santos, A. L., \& Rigotto, R. M. (2011). Território e territorialização: incorporando as relações produção, trabalho, ambiente e saúde na atenção básica à saúde. https://www.scielo.br/j/tes/a/H5BtBJTGvQZgSXKvNrTKphp/?format=pdf\&lang=pt

Souza, G. J., Gomes, C., \& Zanetti, V. R. (2020). Estratégia Da Saúde Da Família: A Dimensão Articuladora Do Território. https://online.unisc.br/seer/index.php/barbaroi/article/view/14643/8865

Souza, J. V. G. d., Friestino, J. K. O., \& Fonsêca, G. S. (2019). Geografia E Saúde Na Graduação Em Medicina: Vivências De Territorialização Na Atenção Primária À Saúde. Revista Baiana de Saúde Pública. https://rbsp.sesab.ba.gov.br/index.php/rbsp/article/view/2844/2809

Souza, M. T. d., Silva, M. D. d., \& Carvalho, R. d. (2010). Revisão integrativa: o que é? Como fazer isso? https://www.scielo.br/j/eins/a/ZQTBkVJZqcWr TT34cXLjtBx/?lang=en 